\title{
The LMS-GT Instrument - A new perspective for quantification with the LIMS-TOF measurement technique
}

Received 00th January 20xx, Accepted 00th January 20xx DOI: $10.1039 / \times 0 \times x 00000 x$

\author{
Reto Wiesendanger ${ }^{a, b}$, Valentine Grimaudo ${ }^{a}$, Marek Tulej ${ }^{a}$, Andreas Riedo ${ }^{a, c}$, Rustam Lukmanov ${ }^{a}$, \\ Niels Ligterink ${ }^{a}$, Rico Fausch ${ }^{\mathrm{a}}$, Herbert Shea ${ }^{\mathrm{b}}$ and Peter Wurz ${ }^{\mathrm{a}}$
}

\begin{abstract}
In this contribution we present the design and first measurement results obtained with a new high-performance laser ablation and ionisation (LIMS) mass spectrometer for solid sample analysis named "LMS-GT", combining high mass- and high spatial resolving powers. The instrument consists of a fs-laser ablation ion source coupled to a time-of flight (TOF) mass spectrometer that provides measurements with a mass resolution $(\mathrm{m} / \Delta \mathrm{m})$ of $10^{\prime} 000$ at full width half max and more over a wide mass range. This resolution enables the separation of the most important isobaric interferences between clusters, molecules and multiple charged ions. Thereby it enables significant improvements of the quantitative analysis of complex samples with the LIMS-TOF technique. The instrument performance is demonstrated by analysis of measurements conducted on various NIST standard reference materials (SRMs). Using these samples we determined detection limits in the ppm range and below, and relative sensitivity coefficients (RSCs), in the range between 0.1 and 10 .
\end{abstract}

\section{Introduction}

The determination of the element and isotope composition of solid samples is of utmost importance in many fields of science and technology. Often optical spectroscopic techniques like $\mathrm{X}$ Ray Fluorescence, Raman Spectroscopy and Laser induced Breakdown Spectroscopy (LIBS) are used. However, mass spectrometry typically provides a higher sensitivity and the possibility of determination of isotope ratios. These key capabilities make mass spectrometry an indispensable tool in analytical chemistry. A wide range of mass spectrometric technologies such as secondary ion mass spectrometry (SIMS) glow discharge mass spectrometry (GD MS) and laser ablation inductively coupled plasma mass spectrometry (ICP-MS) are currently used for analytical investigations and their advantages and drawbacks are well-known ${ }^{1-6}$. Some SIMS instruments provide excellent spatial resolution but SIMS quantitative performance suffers from severe matrix effects, and ion implantation $^{7-9}$. ICP-MS has excellent sensitivities to the trace element level but can suffer from fractionation and interactions between the sample, transportation gas and the plasma10-12. GD-MS provides good sensitivity to trace elements but also suffers from gas-sample interactions and is limited to large

\footnotetext{
a. Space Research and Planetary Sciences, Physics Institute, University of Bern, Switzerland.

b. Soft Transducers Laboratory EPFL-LMTS, Ecole Polytechnique Férérale Lausanne, Switzerland

c. Leiden Observatory, Sackler Laboratory for Astrophysics, Leiden University, The Netherlands

† Footnotes relating to the title and/or authors should appear here.

Electronic Supplementary Information (ESI) available: [details of any supplementary information available should be included here]. See DOI: 10.1039/x0xx00000x
}

sample sizes. Recently GD instruments were coupled to lasers to extend the applicability to non-metallic samples ${ }^{6,13}$.

Laser ablation and ionisation mass spectrometry (LIMS) is an attractive alternative to the mentioned techniques because it provides high sensitivities ${ }^{14-16}$, chemical imaging and depth profiling capabilities ${ }^{17-19}$ and is applicable to most solid samples. LIMS instruments belong to the microprobe category, meaning that spatially highly localised element and isotope analysis can be performed, with little or no sample preparation. High-power pulsed lasers needed for ablation combine in a natural way with time-of-flight (TOF) mass spectrometry. These advantages made the LIMS-TOF-microprobe an attractive technique soon after the first laser became available $20-23$ and a series of commercial LIMS microprobes existed in the 1980'ies and 1990 'ies. These instruments were mainly the LIMA $2 \mathrm{~A}$ and the LAMMA 500 and 1000.

\section{Early commercial instruments}

The LIMA 2A Laser Microprobe was manufactured by Cambridge Mass Spectrometry ${ }^{24}$. Although theoretically mass resolutions ${ }^{25}$ of $\mathrm{m} / \Delta \mathrm{m} \sim 2600$ should have been possible, only values between 250 and 750 were reported ${ }^{26}$. The lack of mass resolving power was assigned to the time resolution of the transient recorder (16 ns), space charge effects in the ablation plume, and the duration of the ionisation laser pulse. Like for other LIMS systems of the time, the quantitative performance of the LIMA 2A was limited due to its dependence on matrix matched standards ${ }^{27}$.

The LAMMA instruments series belonged to the analytical instruments commercialized by Leybold Heraeus in the early 
1980ies ${ }^{28}$. While in LAMMA 500, thin samples are irradiated from the back ${ }^{29}$, LAMMA 1000 allowed to focus the $266 \mathrm{~nm}$ $\mathrm{Nd}$ :YAG laser on the sample in a $30^{\circ}$ angle configuration 30 to spots of 2-15 $\mu \mathrm{m}$ in diameter ${ }^{31}, 32$. The LAMMA 500 instrument includes an optical microscope with incident, transmitted and phase contrast illumination ${ }^{32}$ and an optical resolving power of $\sim 1 \mu \mathrm{m}$. The LAMMA 1000 instrument features an optical microscope with $\mathrm{NA}=0.22$, and a nominal resolving power $0.5 \mu \mathrm{m}^{24}$. However, some authors judged the performance of the microscope as "poor" and rather close to $2 \mu \mathrm{m}^{32,} 34$. Detection limits of $\sim 1 \mathrm{ppm}$ (weight fraction, wf) were reported for the LAMMA instruments ${ }^{30}$, but the need of empirically determined relative sensitivity coefficients (RSC's) rendered quantification difficult.

From today's perspective one can conclude that the early commercial LIMS-TOF instruments lacked quantitative performance due to a combination of

the laser systems of the time subjected to strong matrix effects during ablation and ionisation,

the mass resolving power that was barely sufficient for the resolution of isobaric interferences between elemental ions, molecules, clusters and multiple charged species ${ }^{23,29}$.

slow data acquisition systems for the recording of ion signals with ns duration.

Because of the lack of quantitative performance, the interest in these instruments faded at the beginning of this century and LIMS was replaced by other analytical techniques.

\section{Current state of the art}

Meanwhile, laser- and computer technology continued to evolve quickly: Highly stable, femtosecond lasers opened new horizons in laser ablation and ionisation measurement techniques. Modern computers allow to precisely simulate ion optical systems and design instruments with a degree of complexity that was unreachable at the end of the last century. Today's computers also allow for data acquisition, storage and processing at a speed that was impossible to reach when the first LIMS instruments were commercialised. Some academic groups $^{14,35-38}$ and companies ${ }^{39}$ continued to push the limits of the LIMS technique, often as prototypes for space borne instruments ${ }^{39-43}$.

\section{Spatial resolving power}

Nowadays, lateral resolutions and targeted analysis of locations of interest in the $\mu \mathrm{m}$ range were reported by several groups ${ }^{17}$, 37, 44-46. Kusnetzov et al. reportedon an instrument with lateral resolving power of $75 \mathrm{~nm}$ using an EUV laser with $46.9 \mathrm{~nm}$ wavelength and vertical resolutions of $20 \mathrm{~nm}$ were achieved ${ }^{47}$, 48. Grimaudo et al. and Cedeño et al. reported on analysis conducted vertical resolutions in the $\mathrm{nm}$ range using a fs-IR laser combined with a beam shaper providing hat-top laser profiles ${ }^{49}$ 50.

\section{Mass resolution and reduction of isobaric interferences}

For quantification the resolution of isobaric interferences between elemental ions, oxides, molecules, clusters and multiple charged ions is of utmost importance. The most straight forwards solution to this problem would be to build an instrument with sufficient mass resolving power. For the baseline resolution of most isobars between ions, oxides, clusters and multiple charged ions a mass resolving power (at full width half $\max , \mathrm{FWHM}$ ) of $\mathrm{m} / \Delta \mathrm{m}=10^{\prime} 000$ would be sufficient, while the resolution of elemental isobars would require a resolving power of $m / \Delta m=100^{\prime} 000^{52}$.

However, to our best knowledge, the mass resolution even of large laboratory time of flight instruments is limited to a few thousand $38,46,53,54$. The University of Chicago built a series of highly sophisticated TOF instruments for the analysis of submicron dust particles and surface analysis ${ }^{47,55}$. Stephan et al. report on an instrument of the series that is capable to resolve isobaric interferences between elements with resonant ionisation of neutrals using a sophisticated timing scheme of laser pulses and electrode voltages ${ }^{38}$. A similar approach is taken by Anderson et al. to resolve element isobaric interference between ${ }^{87} \mathrm{Rb}$ and ${ }^{87} \mathrm{Sr}$ with the goal to date lunar minerals 56 . The instrument employs a multi-reflection TOF architecture and the group successfully demonstrated dating of a Martian meteorite ${ }^{44}$. Other multi-reflection instruments were also proposed to extend the time of flight and thereby the mass resolving power ${ }^{57,58}$. Several groups reported on instruments using electrostatic sector fields to extend the time of flight and focus ions in space and time. Instruments with remarkable S- Oand 8- shaped geometries were built and, for example, successfully employed for the characterisation of aerosol particles ${ }^{36,59-61 .}$

Recently, Sysoev proposed a wedge-shaped ion mirror for focussing ions from laser ablation without prior acceleration $^{62}$.Despite the large efforts, none of the mentioned instruments featured sufficient resolving power for baseline resolution of isobars. Arevalo et al. followed a different approach and used an orbitrap mass analyser combined with a pulsed laser source for the analysis of solid samples ${ }^{63}$. The analyser featured a mass resolving power of more than 100 '000. However, space charge limits the number of ions that can be loaded into the orbitrap analyser and thereby its sensitivity and dynamic range. Another approach was taken by Huang et al. who successfully managed to resolve isobars between multiply charged ions from other species exploiting differences in the initial kinetic energies with an orthogonal TOF mass spectrometer ${ }^{53}$. Despite of considerable progress in the design of TOF ion optics with grid-less ion mirrors 64,65 , to our best knowledge no one has so far succeeded to resolve all relevant interferences simultaneously in a laser ablation mass spectrum.

\section{Quantification}

The problems with quantification due to matrix dependence itself were overcome with arrival of new femto-second laser pulses that provide power densities in the $T W / \mathrm{cm}^{2}$ regime. Relative sensitivity coefficients close to one, a pre-requisite for standard-less quantitative analysis, became feasible ${ }^{14,66,67}$. It 
was also demonstrated that detection limits in the sub-ppm range are nowadays possible ${ }^{14,68}$.

\section{Precursor instruments at the University of Berne}

Many of the above-mentioned improvements were accomplished using the LMS (Laser Mass Spectrometer) instrument at the University of Berne ${ }^{16,41}$. The instrument is a small prototype for a space-borne application on planetary surfaces. LMS was initially developed for a Mercury lander mission and since then steadily improved over more than a decade. At the current state of development, the main limiting factor for quantification with the LMS instrument are the isobaric interference of clusters, molecules, singly and multiply charged ions ${ }^{69,70}$. The instrument's resolving power is limited by the miniaturised ion optics, initially developed to fit on a Mercury lander, where volume, weight, and maximum power consumption are very limited. The ion optical system of a new LIMS instrument that is dedicated to laboratory applications would not be restricted by these boundary conditions and could overcome the intrinsic limitations of the miniature mass analyser.

Using the above mentioned progress in the LIMS field, especially the experiences gained with the LMS instrument, such a laboratory instrument was developed over the past four years. To reflect this advance in technological development, the new instrument was named "Laser Mass Spectrometer Gran Turismo" with acronym LMS-GT.

The LMS-GT is designed to fill the gap of high mass- and high spatial resolving power, matrix independence, detection sensitivities in the ppm range and below and the capability to acquire mass spectra over the full mass range in a fraction of a second. These requirements cannot-or only partially - be filled by alternative techniques.

\section{The LMS-GT Instrument}

\section{Requirements}

The most straight forward solution to overcome the problem of isobaric interferences, while providing highest sensitivity over a wide mass range, is a TOF instrument with sufficient mass resolving power. To achieve this goal, a mass resolving power of $\mathrm{m} / \Delta \mathrm{m}>10^{\prime} 000$ at $\mathrm{FWHM}$ is required ${ }^{52}$. To provide best quantitative performance and measurement sensitivity, all ions produced in the ablation plume shall be transmitted from the point of ablation to the detector. With this requirement no instrument fractionation and maximal ion collection efficiency is obtained. An energy transmission bandwidth of $100 \%$ for ions ranging from about $0 \mathrm{eV}$ up to $>180 \mathrm{eV}$ initial energy should provide optimal quantitative performance ${ }^{71-73}$.

The spatial resolution of the LMS-GT instrument should be in the micrometre range to analyse inclusions in meteoritic samples, fossilized bacteria ${ }^{45,69}$ and interstellar grains. Further applications of our instrument for the analysis of materials used in the semi-conductor industry are also considered ${ }^{17,74}$. An insitu microscope camera system should provide online vision on the sample at any time during the analysis to target specific areas on the sample with micrometre accuracy.

\section{Description of the Instrument Layout}

The final instrument design of the TOF mass spectrometer includes a flight path folded twice by ion mirrors, a laser ablation ion source, and a microscope camera system. The SIMION ion trajectory modelling package was used to design and optimize the ion optical system. The ZEMAX optical modelling package was used for the laser and microscope optics design. The mechanical design and analysis was performed in CATIA. Several custom made pre- and postprocessors running on MATLAB allowed the detailed analysis of the simulated ion packets. The mechanical parts were partially manufactured inhouse or by specialized external suppliers. The CAD model of the final instrument, including key assemblies, is depicted in Figure 1.

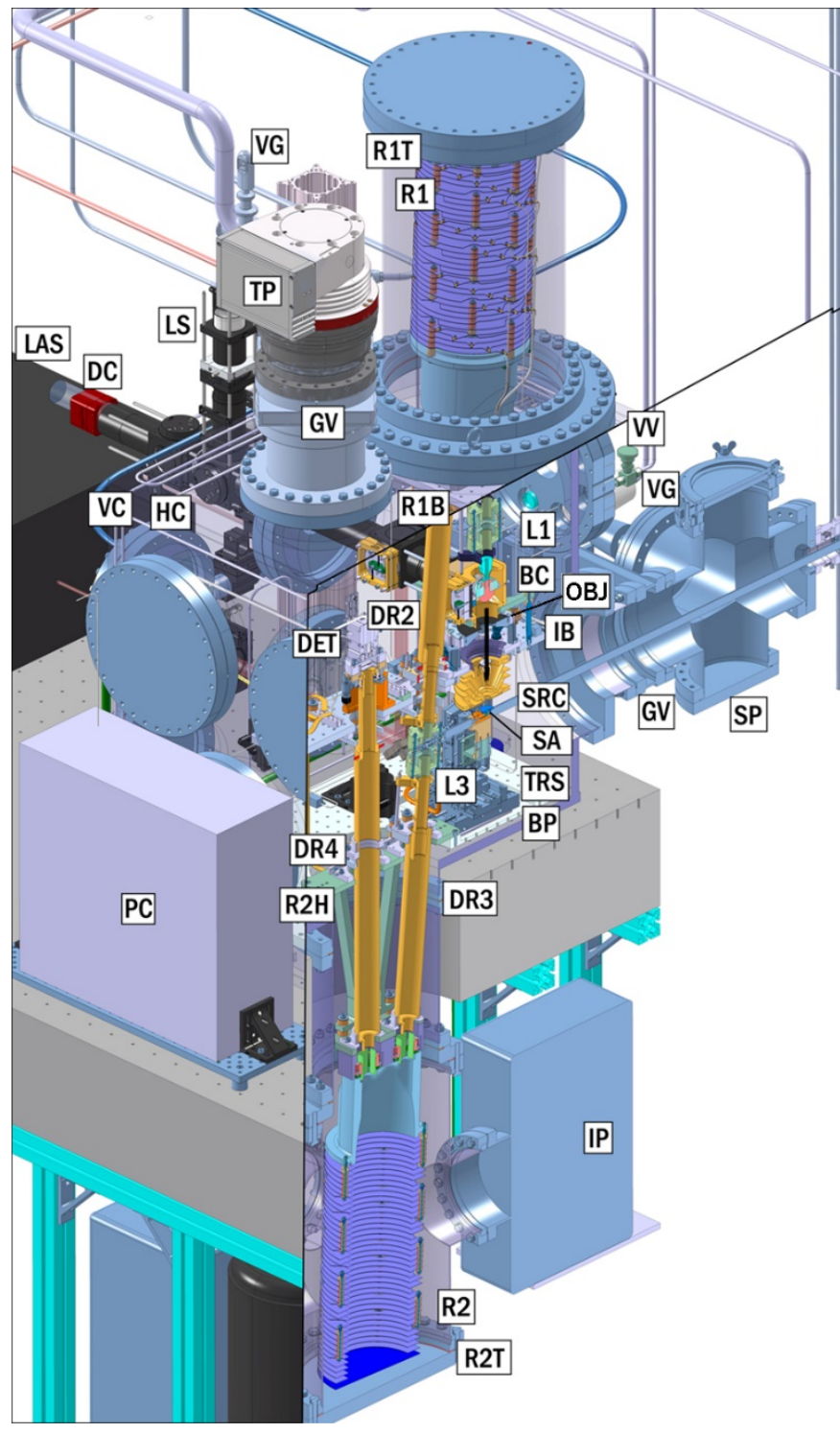

Figure 1: CAD Drawing of the LMS-GT instrument. See text for explanation of the labels. 
The sample (SA) is placed on a three axis translation stage (Physik Instrumente, Germany) supporting sub-micron accuracy in the position adjustment. The laser is focused onto the sample variations in the ambient atmospheric pressure. The mechanical instrument layout is structured in three horizontal plates. Above the baseplate, the instrument bench (IB) and ion mirror bench

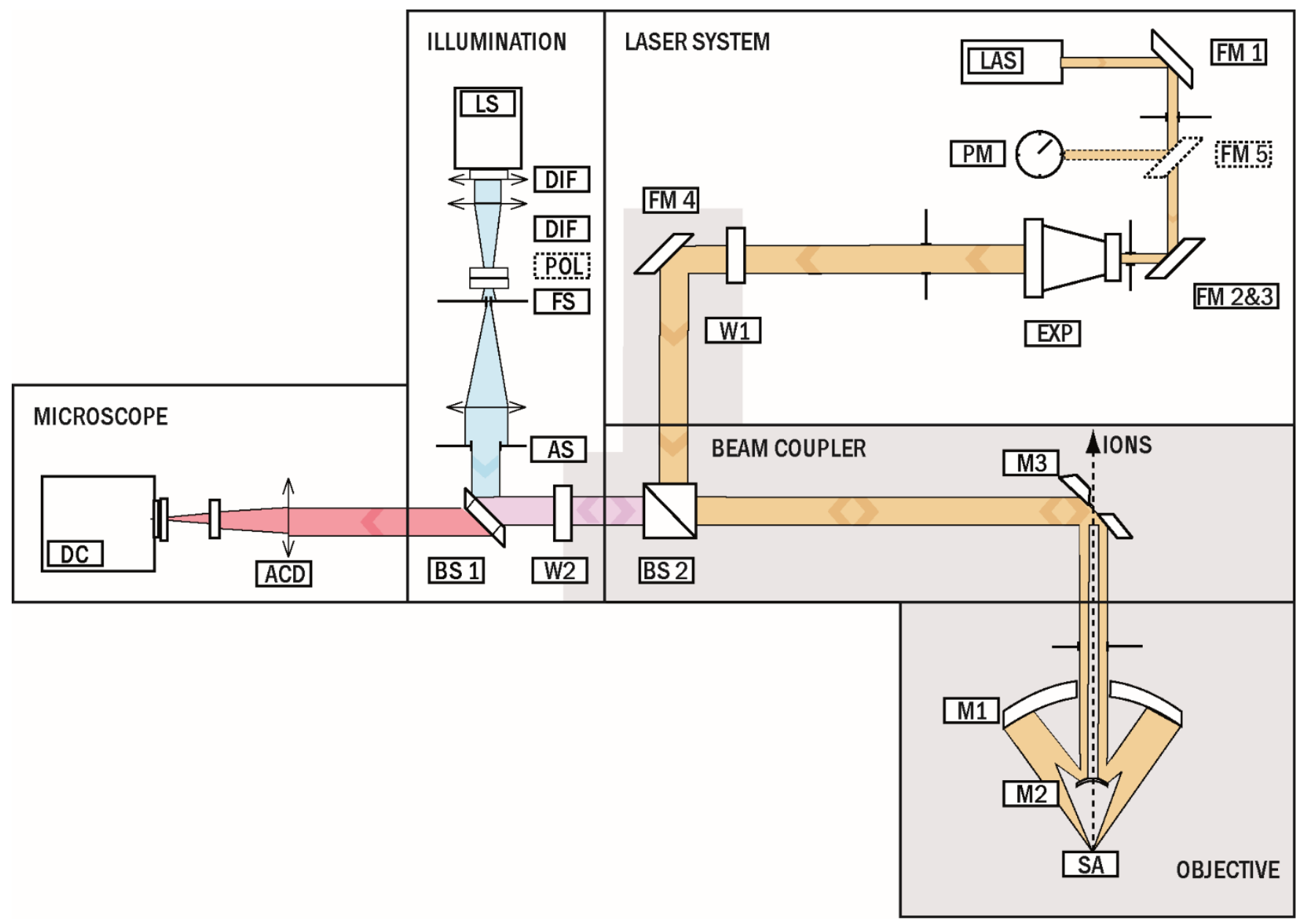

Figure 2: Layout of the microscope and laser optics of the LMS-GT instrument. (See text for explanation of the labels)

by a two mirror objective (OBJ). In the ion source (SRC), the ablated ions are accelerated through the central apertures of the two objective mirrors. Subsequently, the ions reach the Einzel Lenses L1 and L2 (not visible on the picture) where the ion-beam is geometrically re-focused and injected into the first ion-mirror (R1). After leaving the first ion mirror, the ions are guided through the drift tube, through a hole in the table to the second ion mirror (R2) located underneath the optical table. The last part of the drift tubes (DR4) protects the ion packets on their travel to the detector (DET).

The complete instrument, including vacuum chamber laser and optics, is mounted on a $1.3 \times 2 \mathrm{~m}$ sized granite optical table standing on four Newport I-2000 vibration insulators. The table has a hole with $400 \mathrm{~mm}$ diameter that is used to extend the drift length. Apart from the internal cables, the only mechanical connection of the instrument to the chamber are four spacers between the baseplate (BP) and the main vacuum chamber (VC) floor. The size of the baseplate is $600 \times 600 \mathrm{~mm}$ and the spacers are located at the corners. This allows to effectively mechanically de-couple the instrument from mechanical stress and misalignment introduced to the vacuum chamber by
(R1B) provide mechanical interfaces to all other assemblies. All three plates are precision manufactured aluminium plates with $20 \mathrm{~mm}$ (BP) or $12 \mathrm{~mm}$ (IB, R1B) thickness, respectively, and a flatness better than $0.2 \mathrm{~mm}$. The vertical support is provided by 1.5" stainless steel rods. The instrument bench provides precision manufactured (less than $0.02 \mathrm{~mm}$ in tolerance) alignment features for all ion, microscope- and laser optical components.

\section{Vacuum System}

The main vacuum chamber is a customized Pfeiffer "Trinosline" cubic chamber with a volume of $750 \times 750 \times 750 \mathrm{~mm}^{3}$. A total of 13 ports with dimensions between DN CF 160 and CF400 provide the possibility to mount the required windows, feed-through, pumps and extensions. The main extensions consist of a tube with $300 \mathrm{~mm}$ diameter that accommodates R1 (R1T) and a six way cross piece with $300 \mathrm{~mm}$ in diameter and 4 DN 160 CF ports that hosts R2. The chamber is pumped with a water-cooled Pfeiffer HiPace 700 turbomolecular pump (TP) that can be separated from the main chamber with a gate valve (GV) from VAT. To reduce mechanical vibrations introduced by the 
turbomolecular pump, one can switch to two Agilent Starcell 300 ion getter pumps (IP). The additional advantage of using the ion getter pumps is the reduction of the electronic noise in the system.

A sample introduction port (SP on Figure 1), featuring a DAPP sample transfer arm purchased from UHV design allows quick exchange of samples. Its central part is a DNCF 160 six way cross piece that is pumped by a Pfeiffer HiPace 80 turbomolecular pump.

A bake out system allows to heat all parts of the instrument.
An adjustable mirror support is therefore necessary to compensate the mechanical manufacturing errors and adjust mirrors after installation. Because the system will be operated under vacuum (vacuum parts are shaded in Figure 2), a piezoactuated, vacuum compatible (PI Instruments Piezzo Mike N470) system was designed and manufactured. The motors provide a total of three translational and two rotational degrees of freedom. The laser light is delivered to the objective through a series of fold mirrors (FM 1- 4). A beam expander (EXP) from Altechna expands the beam to the diameter of M2. M3 is a

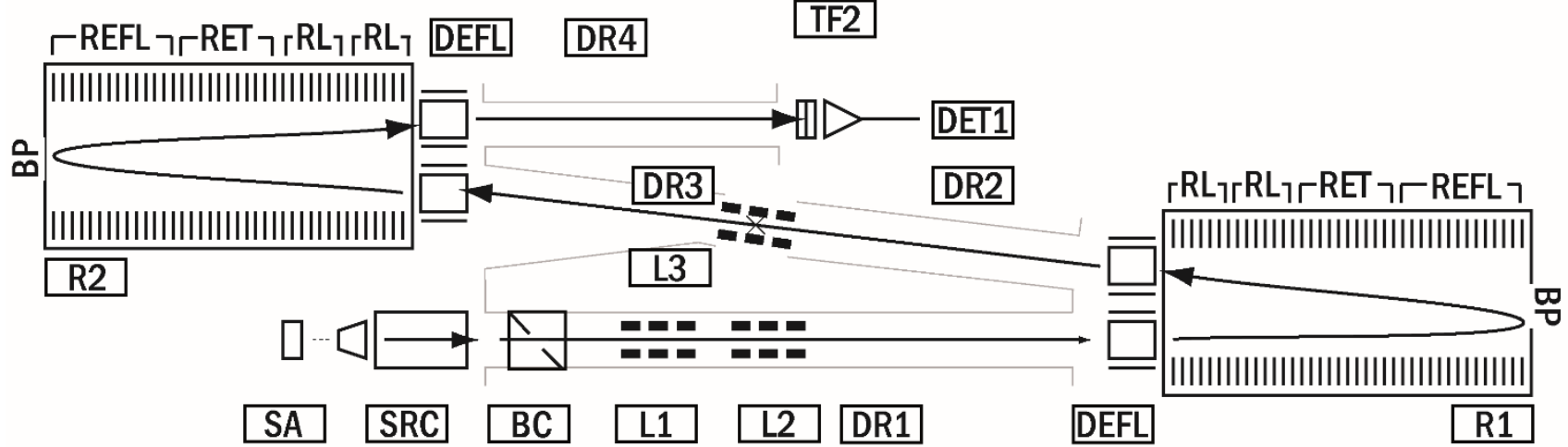

Figure 3: Layout of the ion optics of the GT instrument (See text for explanation of the labels)

Fourteen heating cords (HC, HTC452003, BriskHeat) with a total power of $3080 \mathrm{~W}$ are located on the air side walls of the vacuum chamber. The heating cords are wired to three independent closed-loop controlled circuits. The vacuum side part of the instrument on can also be temperature controlled with three additional vacuum compatible heating circuits located at BP, IB and R1B. All temperatures can be set and monitored individually in a dedicated unit in the control electronics rack. Usually the chamber and the instrument are baked out at $80^{\circ} \mathrm{C}$ for two days followed by 1-2 days of cooling phase prior to measurements. If temperature sensitive materials are investigated, the vacuum can also be established without the bake-out, but requires more time.

\section{Microscope optics and Laser System}

The light optical system, schematically represented in Figure 2, plays a central role in the instrument. Its primary purpose is to guide the pulsed laser beam from the source (LAS) to the objective (OBJ), where it is focused on the sample (SA) to a spot of 1-2 micrometres in diameter, depending on the laser wavelength. A Ti-Sappire laser system from Clark MXR is used to produce femtosecond laser pulses (pulse width of about 190 fs $\lambda=775 \mathrm{~nm}$ ) ${ }^{14}$. The laser's pulse repetition rate is up to $1 \mathrm{kHz}$ and the maximal output pulse energy is $1 \mathrm{~mJ}$.

The objective consists of a two mirror system. The large, primary mirror $\mathrm{M} 1$ has a clear aperture of $82.8 \mathrm{~mm}$ and a mechanical diameter of $110 \mathrm{~mm}$. The central aperture measures $42 \mathrm{~mm}$ in diameter. The small mirror (M2) diameter measures $25 \mathrm{~mm}$ with a clear aperture of $22.5 \mathrm{~mm}$. The central bore has a diameter of $10 \mathrm{~mm}$. To provide optimal performance, $\mathrm{M} 1$ and $\mathrm{M} 2$ need precise alignment with respect to each other. specially designed fold mirror with a central bore that allows the ions to pass through and enter the ion optical system.

An in-line microscope with a resolving power of $2 \mu \mathrm{m}$ allows to verify the laser spot size and location on the sample. This microscope can also be used to target specific sample features during the measurement campaign and uses the same objective as the laser. The microscope and laser light paths are combined inside the vacuum chamber using a dichroic mirror (BS 2) that reflects only the laser's light at $\lambda=775 \mathrm{~nm}$. The light source consists of a tungsten-halogen lamp (Thorlabs QTH10B) and a series of diffusers (DIF), a field stop (FS) and an aperture stop (AS). A 50:50 broad-band beam splitter (BS1, Thorlabs, BSW27) combines the illumination with the image-light path. The image is focussed with an achromatic doublet lens (ACD) and recorded with a CMOS camera (DC, Photonfocus SM1).

\section{Ion Optics}

The ion optical system of the LMS-GT instrument is depicted on Figure 3. The ion optical design was entirely carried out using SIMION. Custom made MATLAB pre- and post- processors were made to create the initial ion populations and to visualize the simulation results. Using the pre-processor, an initial ion plasma plume with a chemical composition representing the National Institute of Standard and Technology (NIST) standard reference material (SRM) 664 high carbon steel material was created. All elements except iron were represented in the correct abundances and isotopic composition. Due to the high abundance, the iron content was reduced by a factor of 1'000 to accelerate the calculations. The initial velocity distribution $f(v)$ was calculated as drifting Maxwell-Boltzmann distribution according to ${ }^{71,75}$ 


$$
f(v)=c v^{2} e^{-\frac{m(v-u)^{2}}{2 k_{B} T}}
$$

Equation 1

with $v$ the velocity of the ion, $k_{B}$ the Bolzmann constant. The temperature $(T)$ and the mean velocity $(u)$ were correlated to measurements performed on the LMS prototype instrument and adjusted so that the initial kinetic energies for all elements in the simulated population was limited to a maximum of 180 $\mathrm{eV}^{71-73}$. The angular distribution of ions was calculated according to ${ }^{76}$.

$$
F(\theta)=F(0)\left(1+\tan ^{2} \theta\right)^{\frac{3}{2}} /\left(1+k^{2} \tan ^{2} \theta\right)^{-\frac{3}{2}}
$$

Equation 2

The value for $k=1.4$ was determined from the literature ${ }^{77}$ and $\theta$ is the emission angle of the ion. The preliminary SIMION analysis showed that the drift length should be longer than $4 \mathrm{~m}$ to achieve the desired mass resolving power. To accommodate this length in the available facility, the flight path had to be folded twice. Figure 6 shows a schematic view of such a design. The ions ablated from the sample (SA) enter the ion source (SRC) and are accelerated to $5 \mathrm{kV}$ drift potential. Subsequently, a series of five $\mathrm{V}$-shaped electrodes serve as electrostatic lenses to accelerate and focus the ions into a parallel beam. After passing through the beam coupler (BC), the ion beam is further focussed by two lenses ( $L 1$ and L2) before entering the first ion mirror (R1). The ion mirror is composed of 40 ring-shaped electrodes with $140 \mathrm{~mm}$ inner- and $200 \mathrm{~mm}$ outer diameter. The ion-mirror is closed by a back-plate electrode (BP). Similar to a previously used design 65,78 , the ion mirror electrodes are grouped into four segments. The two segments close to the entrance work as lens (RL), the next section serves to slow down the ions (RET) before the ions are reflected into the last ion optical section (REFL). Both ion mirrors have the same architecture and are linked with a drift tube with $50 \mathrm{~mm}$ in diameter. The drift tube is equipped with $25 \mathrm{~mm}$ apertures at the entrance and exit and an electrostatic lens in the centre (L3). At the same location, there is a first time focus with mass resolutions of $m / \Delta m>1600$. It is intended to use this location for the placement of a blanking pulser in the future ${ }^{15,65}$. The first (DR1) and the last (DR4) drift tubes are vertically mounted. The intermediate section (DR3) is tilted by $5^{\circ}$. Both ion mirrors are inclined by $2.5^{\circ}$ with respect to the ion optical axis of the ion source electrodes to deflect the ions into the V-shaped trajectories. To cope with mechanical misalignments, each ion mirror possesses four deflection plates (DEFL) at the entrance and exit. Overall, the ions drift over a distance of $4.3 \mathrm{~m}$. All ionoptical elements, except the electrodes of the ion mirrors, are gold plated. The electrodes of the ion mirror are - like the majority of the other instrument parts- Alodine 1200-coated to provide an optimal electrical conductivity.

For the detection of the ions, we use a MagneTOF detector from ETP. According to the manufacturer, the detector has a peak width for a single ion event of $550 \mathrm{ps}$. Using our measurement computer equipped with an Acquiris U1084A acquisition card with 8 bit vertical resolution, sampling rate of up to $4 \mathrm{GS} / \mathrm{s}$ and
1.5 $\mathrm{GHz}$ analogue bandwidth, we determined a peak width for single ion detection between 700 and 800 ps depending on the detector operation voltage. The detector is mounted on a kinematic mount that allows manual, horizontal alignment of the detector.

\section{Materials and Methods}

\section{Samples}

We used the NIST SRM 661 (AISI 4340), SRM 664 high carbon steel and SRM 665 electrolytic iron to characterize the LMS-GT instrument. This allows a direct comparison of the results to previous measurements on other instruments ${ }^{14}$. The steel samples were provided in the form of rods with $4 \mathrm{~mm}$ diameter. Sections of about $3 \mathrm{~mm}$ height were glued on a stainless steel sample holder and finally grinded with a diamond file to remove ablation craters and residuals of precedent measurement campaigns. No further sample processing or preparation was performed on any of the samples.

We used the NIST SRM $981(\mathrm{~Pb})$ and SRM $986(\mathrm{Ni})$ with certified isotope abundances for the determination of isotope ratios. The SRM 981 comes in the form of a wire that was directly mounted as received on the sample holder. The SRM 981 was supplied as a powder that was pressed into a pellet of $5 \mathrm{~mm}$ diameter and $1 \mathrm{~mm}$ thickness. The samples were attached to the stainless steel holder using ultra high vacuum copper tape. The mass resolution was measured at full width half $\max (\mathrm{FWHM})$. We also measured the relative isotope ratios of $\mathrm{K}$ and $\mathrm{W}$ on a Tungsten-Rhenium wire that was obtained from a decommissioned electron emitting YO filament from Kimball Physics used in another instrument ${ }^{79,} 80$

For testing the spatial resolving power we acquired mass spectra on a USAF 1951 negative resolution test target. The target was purchased from Thorlabs (R1DS1N). It consists of a $1^{\prime}$ diameter lime soda glass, with a $0.12 \mu \mathrm{m}$ thick chrome layer. The test target's line patterns were removed from the chrome layer using a photolithographic process. Thus, the lines are transparent while the surroundings remain black. We performed our test measurements on element 1 from Group 7 of the target. The line width of these features is $3.931 \mu \mathrm{m}$.

\section{Measurement Procedure}

Relatively large voltage values are applied for the ion optics when the instrument is operated at the optimal performance. During commissioning of the instrument, reduced voltages were calculated in SIMION and then applied to the instrument. Mass spectra of ions were acquired from the first laser shot, proving that the mechanical alignment and the calculation were made sufficiently accurate. Subsequently, the voltages were gradually ramped up to the nominal drift potential of $5 \mathrm{kV}$. During the ramp-up phase, test spectra on a Ti foil purchased from Goodfellow were acquired. No further optimisation of the instrument parameters was performed later on except the laser 
power and detector voltage to optimize the signal to noise ratio. For the convenience of the operator, the microscope camera was aligned to centre the ablation crater in the image.

The conversion from a TOF spectrum to a mass spectrum, the peak area integration and calculation of the isotope ratios was performed according to Meyer et al. 81 The conversion from time- to mass spectrum is performed according to Equation 3.

$$
\frac{m}{z}=C *\left(t_{0}-t\right)^{2}
$$

Equation 3

$C$ and $t_{0}$ are calibration constants found with a linear regression of the square root of Equation 3 using 17 peaks with $\mathrm{m} / \mathrm{z}$ below or equal 52 as input. With this mass calibration, the mean residual of the mass calibration is $1.8 \cdot 10^{-5}$. If peaks with $\mathrm{m} / \mathrm{z}$ ratios above 56 were considered, the quality of the mass calibration decreased, because the highly abundant Fe element distorts the baseline and thereby the accuracy of the mass calibration. A manual mass calibration with ${ }^{10} \mathrm{~B}$ and ${ }^{208} \mathrm{~Pb}$ as reference peaks showed similar results, so that an error introduced by the least square algorithm can be excluded.

\section{Results}

\section{Spatial Resolving Power}

Figure 4 shows the surface of the USAF $1951^{82}$ resolution test target in the region of group 7, element 1 , after recording of 28 single mass spectra at a laser energy of $1.2 \mu \mathrm{J} /$ shot (measured at the sample surface). The sample was displaced by $1 \mu \mathrm{m}$ perpendicular to the line pattern after each laser shot. Thus a total distance of $28 \mu \mathrm{m}$ was chemically mapped. The track of the laser ablation can be seen as a metallic line on Figure 4, taken with a laboratory brightfield microscope from Zeiss. The zone of ablation can be identified in the centre of the track. It is surrounded by a larger affected zone. The recorded peak intensities of all four $\mathrm{Cr}$ isotopes are shown on the right side of Figure 4. A clear decrease of the signal intensity of all
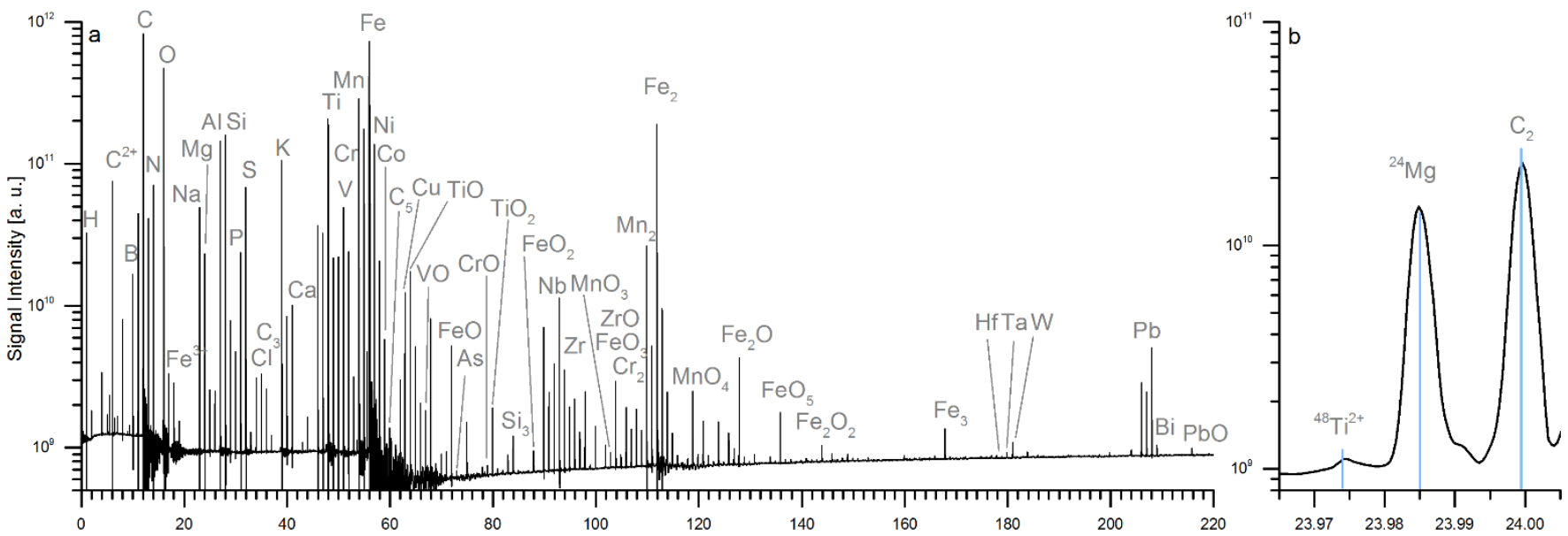

Figure 5: Panel a: Accumulation of 20'000 spectra acquired on the NIST SRM 664 Panel b: Zoom in the region of $\mathrm{m} / \mathrm{z}=23.96$ and 24.01 . The blue lines indicate the theoretical locations of the peak centres.

isotopes can be observed when the laser crosses the lines, negative pin. The voltage was chosen to bring the signals from 
minor and trace elements above the noise level. All detected elements, as well as the major clusters, molecules and multiple charged ions are labelled. In the section with $\mathrm{m} / \mathrm{z}>100$, many minor clusters are present and not all are labelled for the clarity of the figure.

The mass spectrum shown on Figure 5, was filtered with a wavelet thresholding-based approach but the mass spectrometric analyses were conducted on the raw data. ${ }^{85} \mathrm{~A}$ stationary wavelet transformation (MATLAB wavelet toolbox ${ }^{\mathrm{TM}}$ ) provided a six level decomposition of the time signal using the biorthogonal wavelet (BIOR 2.2). A peak-free region at the beginning of the measurement provides the variance of the background signal, which we used to empirically select the level-dependent thresholds. Soft shrinkage ${ }^{86}$ of the according coefficients led to the smoothed time signal, which was then converted into a mass spectrum. All analysis subsequently shown was performed on the raw data.

\section{Mass resolving power}

For the investigation of the mass resolving power, measurements were conducted on the $\mathrm{Ti}$ foil after full commissioning and optimization of the resolving power of the instrument. To obtain the spectrum shown in the supplementary material, the Ti foil was irradiated with 1.2 $\mu \mathrm{J} /$ pulse (measured at the sample surface) and 60 mass spectra were accumulated. The mass resolution is calculated using the equation $m / \Delta m=1 / 2 t / \Delta t$ used in TOF mass spectrometry. The mass peak centre $t$ was determined from the maximum peak value and $\Delta t$ is the measured full width half maximum (FWHM). The SNR was calculated as the ratio of the RMS noise level and the signal amplitude. The results of the analysis are summarized in Table 1.

Further studies of the mass resolving power were conducted on the NIST SRM 664 (Figure 5). A zoom into the section between $\mathrm{m} / \mathrm{z}=23.96$ and 24.015 is shown in Figure 5, panel b. It demonstrates the instrument's mass resolving power, and ability to resolve isobaric interferences between molecules, clusters, singly and multiply charged ions. Three peaks in the vicinity of $\mathrm{m} / \mathrm{z}=24$ are shown. The two larger peaks are attributed to ${ }^{24} \mathrm{Mg}^{+}$and the ${ }^{12} \mathrm{C}^{2+}$ cluster. The theoretical mass value of each species is indicated as blue, vertical line in the mass spectrum. The measured mass resolution $m / \Delta m$ at FWHM of the ${ }^{24} \mathrm{Mg}^{+}$and ${ }^{12} \mathrm{C}_{2}{ }^{+}$mass peaks are 8601 and 8998 respectively. The SNR of the ${ }^{24} \mathrm{Mg}$ and ${ }^{12} \mathrm{C}_{2}{ }^{+}$peaks are 288 and 619 respectively. Also a less intense peak can be observed at $\mathrm{m} / \mathrm{z}=23.974$, which is attributed to doubly charged ${ }^{48} \mathrm{Ti}$.

\section{Comparison of simulation to measurement}

The full peak widths of 38 peaks in the range of $\mathrm{m} / \mathrm{z}$ between 1 and 208 were analysed. The measured mass resolution for two measurement campaigns is plotted as red and blue squares on Figure 6 . The blue squares represent an accumulation of mass spectra of $20 \times 10$ laser shots and the red squares an accumulation of $20 \times 1000$ spectra, respectively. The mass resolution determined from the SIMION simulation are represented as yellow circles. For a time-of-flight instrument, the peak width follows a square root function of mass. The yellow curve in Figure 6 panel a, is a square root function fitted to the theoretical values from the SIMION simulation. The measurements shows good agreement in the range of $\mathrm{m} / \mathrm{z}>30$. In panel $b$, the yellow line shows the mean theoretical mass resolution that should be constant over the full mass range. The spread of the theoretical values around the fitted curves is introduced by random sampling of the initial kinetic and starting angle distributions of the generated ions, which will smooth out with more ions being used in the simulations. For a real instrument, the mass resolution can be written as:

$$
R=\frac{m}{\Delta m}=\frac{1}{2} \frac{t}{\Delta t}=K_{1} \frac{t}{\Delta t_{a c q}+\Delta t_{d e t}+\Delta t_{i o}}
$$

Equation 4

Where $\Delta t_{a c q}$ is the broadening due to the finite signal sampling time of the data acquisition card (250ps). $\Delta t_{\text {det }}$ is the minimum detector peak width including the pulse dispersion due to the signal cables, feedthroughs, and the bandwidth of the acquisition system. The value of $\Delta t_{\text {det }}$ was determined to $800 \mathrm{ps}$ using measurements of single ion events. $\Delta t_{i o}$ represents the resolution of the ion optics that is proportional to the time of flight $t . K_{1}$ is an instrument dependent constant. Because $\Delta t_{\text {aca }}$ and $\Delta t_{\text {det }}$ are constant for all values of $\mathrm{t}$, they have a stronger effect on low $\mathrm{m} / \mathrm{z}$ values compared to higher ones. The measured mass resolution gradually reaches a plateau at $\mathrm{m} / \Delta \mathrm{m}$ $\approx 8000$ for the accumulation of 20 mass spectra and at $\mathrm{m} / \Delta \mathrm{m} \approx$ 9000 for the accumulation of $20^{\prime} 000$ mass spectra. This difference can be explained by the effect of the initial crater formation process during which the laser ablation process is less stable ${ }^{68}$. In the second case more spectra are recorded under stable ablation conditions, which results in increased mass resolution. For visualisation, fits of Equation 4 are shown as blue and red curves in Figure 6.

\begin{tabular}{|c|c|c|}
\hline Isotope & Measured Resolution & SNR \\
\hline${ }^{46} \mathrm{Ti}$ & 12480 & 18.2 \\
\hline${ }^{47} \mathrm{Ti}$ & 11787 & 22.5 \\
\hline${ }^{48} \mathrm{Ti}$ & 14692 & 245.6 \\
\hline${ }^{49} \mathrm{Ti}$ & 17199 & 20.9 \\
\hline${ }^{50} \mathrm{Ti}$ & 17998 & 18.2 \\
\hline
\end{tabular}

Table 1. Resolution and SNR measured on Ti foil 


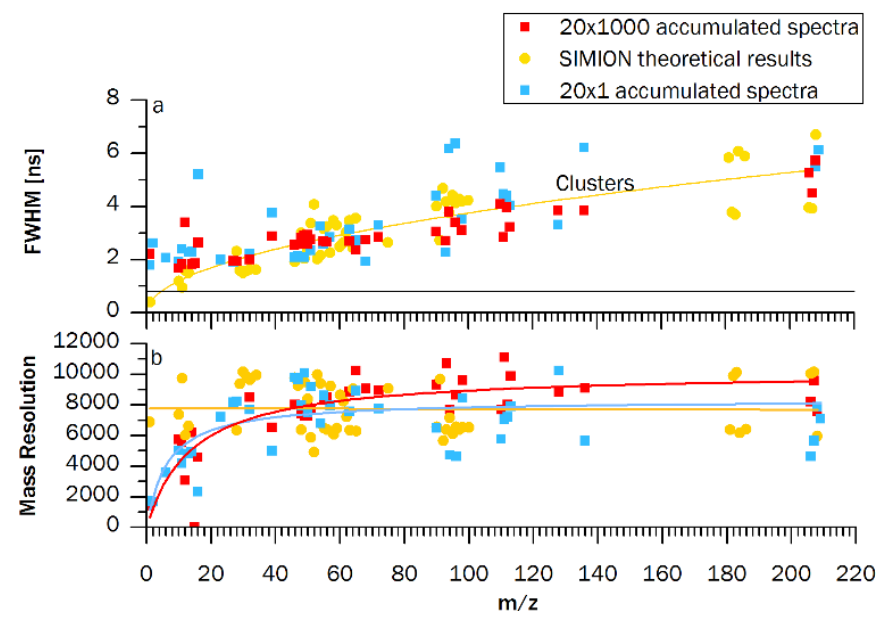

Figure 6: Comparison between theoretical and measured peak width (panel a) and mass resolution (panel b)

Some peaks between $\mathrm{m} / \mathrm{z}=100$ and 140 could be identified in the mass spectra but were not part of the simulations. These peaks belong to clusters or molecules. One example is the mass peak at $\mathrm{m} / \mathrm{z}=112$ which is attributed to ${ }^{56} \mathrm{Fe}_{2}{ }^{+}$. These ions were not present in the initial population of the simulation. On the other hand, some trace elements, for example the isotopes of $\mathrm{W}$, were simulated and identified in the mass spectrum. However, due to low SNR, no sufficiently precise measurement of the mass resolution could be obtained. Therefore these elements are not represented on Figure 6.

\section{Mass Calibration Accuracy}

The mass calibration accuracy for a specific mass peak was calculated as:

$$
a=\frac{m_{-} \text {meas }}{m_{-} t h}-1
$$

Equation 5

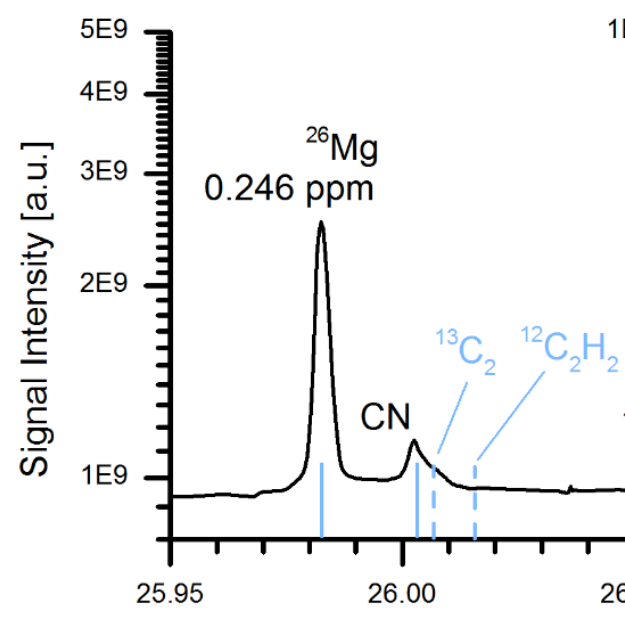

Figure 7: Selection of trace elements from NIST SRM 664 and 665. The abundances are given in atomic fraction. The data shown represents raw data from an accumulation of 20 '000 mass spectra. The blue lines correspond to theoretical values of the assigned mass peaks. ${ }^{13} \mathrm{C}_{2}$ and ${ }^{12} \mathrm{C}_{2} \mathrm{H}_{2}$ are clusters that were sought but not found in the spectrum.
Where $m_{\text {mmeas }}$ is the mass measured at the peak centre and $m_{-}$th is the theoretical mass calculated from values published by the International Union of Pure and Applied Chemistry ${ }^{87}$. The average mass calibration accuracy for singly charged element ions in the mass range between $\mathrm{m} / \mathrm{z}=1$ and 55 is $+18.8 \mathrm{ppm}$. The mean accuracy 56 of the mass scale calibration in the region of $\mathrm{m} / \mathrm{z}>56$ is $-83 \mathrm{ppm}$.

The highly accurate mass calibration allows to precisely identify and quantify mass peaks in the spectrum. On Figure 5, panel b, the region of the spectrum around $\mathrm{m} / \mathrm{z}=24$ is shown. The high resolving power of the LMS-GT allows to clearly separate the elemental ${ }^{24} \mathrm{Mg}^{+}$from the ${ }^{12} \mathrm{C}_{2}{ }^{+}$cluster and doubly charged ${ }^{48} \mathrm{Ti}$. The mass calibration accuracy of better than $5 \mathrm{ppm}$ for the larger peaks and $20 \mathrm{ppm}$ for the ${ }^{48} \mathrm{Ti}^{2+}$ allows to precisely identify the species.

Figure 7, panel a shows how the LMS GT's ability to separate the ${ }^{26} \mathrm{Mg}$ trace isotope peak with an abundance $0.246 \mathrm{ppm}$ (atomic fraction) from surrounding clusters and molecules. The high accuracy of the mass calibration further allows to identify the origin of the cluster. We identified three candidate clusters, ${ }^{12} \mathrm{C}^{14} \mathrm{~N}^{+},{ }^{12} \mathrm{C}_{2} \mathrm{H}_{2}{ }^{+}$and ${ }^{13} \mathrm{C}_{2}{ }^{+}$as potential source for the mass peak found at $\mathrm{m} / \mathrm{z}=26.003$. The theoretical masses of each cluster are marked with blue lines in Figure 7 . The study allowed to clearly identify the $\mathrm{CN}^{+}$molecule as a source of the observed mass peak. The accuracy of the calibration is $-9.6 \mathrm{ppm}$, while it is -4843 and -1407 for ${ }^{12} \mathrm{C}_{2} \mathrm{H}_{2}{ }^{+}$and ${ }^{13} \mathrm{C}_{2}{ }^{+}$respectively.

Figure 7, panel b shows the partially resolved peaks of the ${ }^{80} \mathrm{Se}^{+}$ trace isotope with an abundance of $1.024 \mathrm{ppm}$ (af) and the ${ }^{48} \mathrm{Ti}^{16} \mathrm{O}_{2}{ }^{+}$oxide. The accuracy of the mass calibration for the two peaks are -64.5 and $-571 \mathrm{ppm}$ respectively. From all the elements in the NIST SRM 664, Pb and $\mathrm{Bi}$, have the highest mass. These trace elements and the abundance of their minor isotopes, ${ }^{204} \mathrm{~Pb}$ and ${ }^{209} \mathrm{Bi}$ are shown on Figure 7 . The mass calibration accuracy achieved for these isotopes is -36 and +44 ppm.

Together with the high mass resolving power, the high mass calibration accuracy over the full mass range allows to precisely

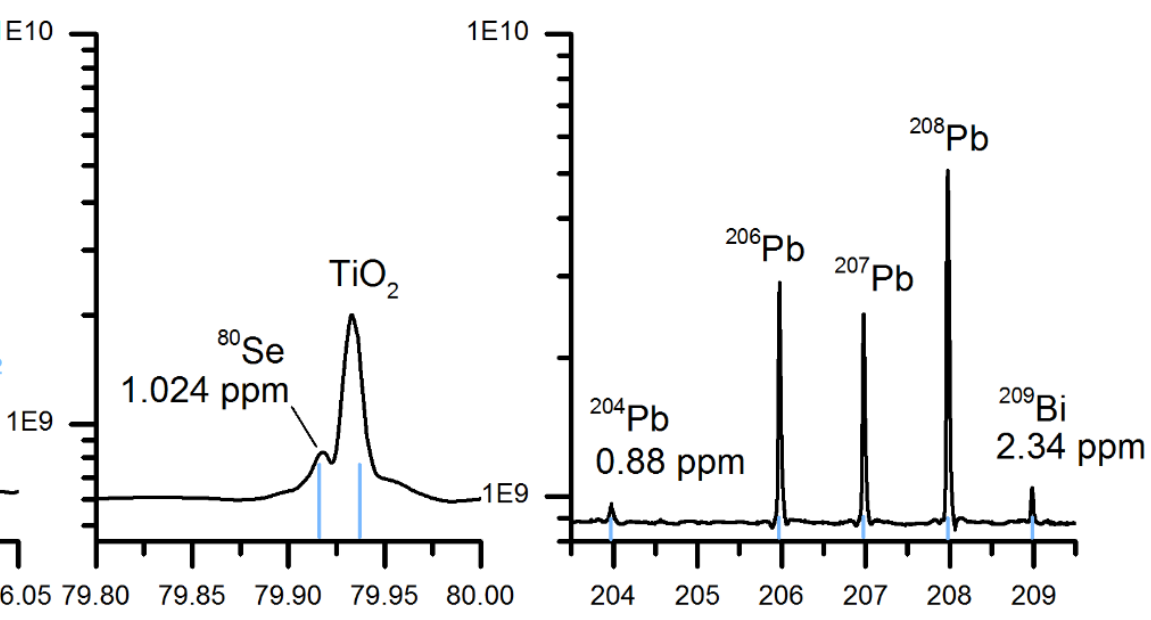

$\mathrm{m} / \mathrm{z}$ 
assign peaks and to quantify them accordingly, which was not possible with previous LIMS-TOF instrumentation with less resolving power.

\section{Dynamic Range}

At the detector gain chosen for the measurements presented in Figure 5, the mass peak of the ${ }^{56} \mathrm{Fe}$ suffers from saturation. Based on the measurement of the signal intensity of minor elements and the known abundances of these elements from the standard, we calculated the saturation level of ${ }^{56} \mathrm{Fe}$ to a factor of 200.

The next smaller peaks are ${ }^{48} \mathrm{Ti},{ }^{55} \mathrm{Mn},{ }^{28} \mathrm{Si}$ and ${ }^{27} \mathrm{Al}$ and appear at SNR between 3922 and 5502. Table 2 gives a selection of the largest and smallest peaks that were identified in the spectrum shown on Figure 5.

\section{Detection Limits}

The trace isotopes shown in Table 2 contain light and heavy elements, as well as metals and non-metals. From all these elements, $\mathrm{Bi}$ represents a suitable trace element and can be used to determine an upper boundary for the detection sensitivity of the LMS-GT instrument. It was measured at an SNR of 2.5 and a concentration of $2.3 \mathrm{ppm}$ (af). The detection limit for this element is therefore in the range of $1 \mathrm{ppm}$. The calculation of SNR and detection limits is the same we used in earlier work ${ }^{81,90}$ Some elements with high relative sensitivity coefficients show even detection limits in the ppb range.

\begin{tabular}{|c|c|c|c|}
\hline \multirow[t]{2}{*}{ Isotope } & \multirow{2}{*}{$\begin{array}{l}\text { Sample } \\
\text { SRM Nr. }\end{array}$} & \multirow[t]{2}{*}{ SNR } & \multirow{2}{*}{$\begin{array}{l}\text { Th. Abundance } \\
\text { [ppm af] }\end{array}$} \\
\hline & & & \\
\hline${ }^{48} \mathrm{Ti}$ & 664 & 5502 & 1924 \\
\hline${ }^{55} \mathrm{Mn}$ & 664 & 4660 & 2472 \\
\hline${ }^{28} \mathrm{Si}$ & 664 & 4285 & 1177 \\
\hline${ }^{27} \mathrm{Al}$ & 664 & 3922 & 161 \\
\hline${ }^{18} \mathrm{O}$ & 664 & 58.2 & 0.118 \\
\hline${ }^{24} \mathrm{Mg}$ & 664 & 384.2 & 1.77 \\
\hline${ }^{25} \mathrm{Mg}$ & 664 & 49.9 & 0.224 \\
\hline${ }^{26} \mathrm{Mg}$ & 664 & 47.5 & 0.246 \\
\hline${ }^{33} \mathrm{~S}$ & 665 & 30.2 & 0.77 \\
\hline${ }^{15} \mathrm{~N}$ & 664 & 6.8 & 0.424 \\
\hline${ }^{10} \mathrm{~B}$ & 665 & 5.4 & 1.36 \\
\hline${ }^{204} \mathrm{~Pb}$ & 664 & 4.2 & 0.88 \\
\hline${ }^{80} \mathrm{Se}$ & 664 & 2.9 & 1.024 \\
\hline${ }^{209} \mathrm{Bi}$ & 664 & 2.5 & 2.34 \\
\hline
\end{tabular}

Table 2: Selection of major, minor and trace isotopes found in NIST SRM 664 and 665. The certified abundances are given in atomic fraction

\section{Relative Sensitivity Coefficients}

For standard free quantitative chemical analysis, relative sensitivity coefficients close to 1 are of crucial interest. Figure 8 depicts the RSCs determined on NIST SRM 664. Because Fe, O and $C$ are subjected to saturation, these elements are not shown and the RSCs were evaluated relative to Ti. The RSCs were calculated according to:

$$
R S C_{x}=\left(\frac{A_{x \text { measured }}}{A_{T \text { i measured }}}\right) /\left(\frac{A_{x \text { standard }}}{A_{\text {Ti standard }}}\right)
$$

Equation 6

Where $A_{x}$ stands for the abundance of element $X$. We determined the RSC from 12 locations on the sample with a pulse energy of $2 \mu \mathrm{J}$ (measured on the sample) to average over spatial material inhomogeneities in the form of micrometre sized inclusions in the SRMs ${ }^{88}$. The majority of elements lighter than iron (marked with solid symbols), shows RSC values within the range between 0.1 and 10, as expected when using an IR fs laser. For Ca an RSC of 16.8 was measured.

Like on previously used MCP detector systems ${ }^{15,89}$, we observed a strong effect of the highly abundant Fe on the detection efficiency of the ETP detector system. Elements with $\mathrm{m} / \mathrm{z}>56$

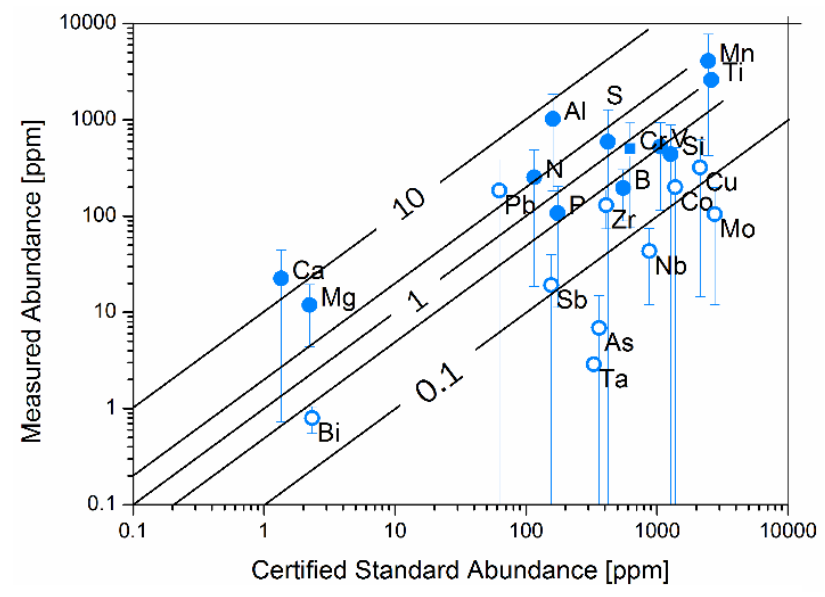

Figure 8 RSC values determined from NIST SRM 664

are detected with reduced sensitivity, thus lower RSCs. We attribute the degradation of the detection efficiency to a temporary depletion of electrons from the dynodes in the detector after the arrival of the high flux of Fe ions. To visualize the impact of this effect, elements are marked with outlined symbols in Figure 8. The RSCs in the high mass region drop to the range of $10^{-2}$. Despite their high masses, some elements like $\mathrm{Bi}$ and $\mathrm{Pb}$ show RSCs close to 1 which is explained by their high ionisation efficiency that compensates for the loss of detection efficiency ${ }^{66}$.

\section{Isotope Abundance Accuracies}

Figure 9 summarizes the results of the isotope accuracy analysis performed on NIST SRM 661, 664, 665, 981 and 986 and the WRe wire. The best isotope ratios achieved in the present measurements are in the per mill range for elements with more than $100 \mathrm{ppm}$ (af) concentration. A positive correlation between the isotope abundance (and thereby SNR) can be observed. However, a large scatter is also present which is attributed to signal ringing after major isotope peaks, which stretches over several mass units in the mass spectrum. Precise 


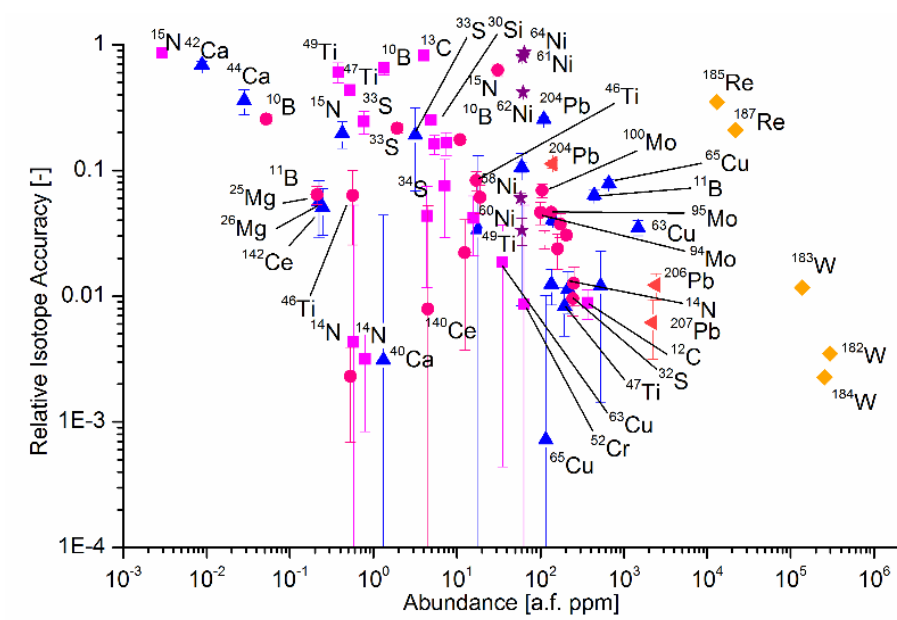

- NIST SRM 664 NIST SRM 665 - NIST SRM 661

WRe Wire « NIST SRM 981 * NIST SRM 986

Figure 9: Isotope ratio accuracies determined from six metallic samples

determination of the peak area windows for the numerical integration is then impeded.

\section{Conclusions}

In the present contribution we introduced a new laser-ablation time of flight mass spectrometer to conduct chemical analysis of solid sample material with mass resolving powers, $m / \Delta m$, of $10^{\prime} 000$. In this first stage of the instrument development, we focussed on the design of the high resolving power ion optics, which proved to work according to the calculations.

In some cases mass resolutions of nearly $18^{\prime} 000$ were achieved. The high mass resolution power allows to resolve most of the isobaric interferences between elemental ions, molecules, clusters and multiple charged species. Together with the high accuracy of the mass calibration in the $10 \mathrm{ppm}$ range, this allows improved quantification using the LIMS technique because mass peaks can be identified unambiguously. The current performance analysis shows trace element sensitivity in the ppm (atomic fraction) range and below. The relative sensitivity coefficients (RSCs) are found in the range of 0.1 to 10 for most light elements. The instrument also shows promising performance to conduct quantitative isotopic analysis with accuracies to the per mill level.

\section{Conflicts of interest}

There are no conflicts to declare.

\section{Acknowledgements}

The authors kindly acknowledge the mechanical and electronic workshop of the University of Bern for the support during the manufacturing and assembly phase._The engineering team of the University of Berne is acknowledged for the kind support and critical reviews during the design phase of the instrument.
This work was supported by the Swiss National Science Foundation SNSF. AR acknowledges the support from the European Union's Horizon 2020 research and innovation programme under the Marie Skłodowska-Curie grant agreement No. 750353

\section{Notes and references}

¥ Footnotes relating to the main text should appear here. These might include comments relevant to but not central to the matter under discussion, limited experimental and spectral data, and crystallographic data.

1. D. Stuewer and N. Jakubowski, Journal of Mass Spectrometry, 1998, 33, 579-590.

J. Pisonero and D. Gunther, Mass Spectrom Rev, 2008, 27, 609-623.

3. E. H. Evans, J. Pisonero, C. M. M. Smith and R. N. Taylor, J Anal Atom Spectrom, 2016, 31, 1057-1077.

4. B. Fernandez, F. Claverie, C. Pecheyran and O. F. X. Donard, Trac-Trend Anal Chem, 2007, 26, 951-966.

5. K. A. Nier, A. L. Yergey and P. J. Gale, <<The >> encyclopedia of mass spectrometry Volume 9 Historical perspectives Part A The development of mass spectrometry.

$6 . \quad$ L. Lobo, B. Fernandez and R. Pereiro, J Anal Atom Spectrom, 2017, 32, 920-930.

7. L. Pillatsch, F. Östlund and J. Michler, Progress in Crystal Growth and Characterization of Materials, 2019, 65, 1-19.

8. F. A. Stevie, R. G. Wilson, D. S. Simons, M. I. Current and P. C. Zalm, J Vac Sci Technol B, 1994, 12, 2263-2279.

9. V. R. Deline, W. Katz and C. A. Evans, Appl Phys Lett, 1978, 33, 832-835.

10. A. Limbeck, P. Galler, M. Bonta, G. Bauer, W. Nischkauer and F. Vanhaecke, Analytical and Bioanalytical Chemistry, 2015, 407, 6593-6617.

11. R. E. Russo, X. L. Mao, H. C. Liu, J. Gonzalez and S. S. Mao, Talanta, 2002, 57, 425-451.

12. D. Kaser, L. Hendriks, J. Koch and D. Gunther, Spectrochim Acta B, 2018, 149, 322-322.

13. M. Tarik, G. Lotito, J. A. Whitby, J. Koch, K. Fuhrer, M. Gonin, J. Michler, J. L. Bolli and D. Gunther, Spectrochim Acta B, 2009, 64, 262-270.

14. A. Riedo, M. Neuland, S. Meyer, M. Tulej and P. Wurz, J Anal Atom Spectrom, 2013, 28, 1256-1269.

15. R. Wiesendanger, M. Tulej, A. Riedo, S. Frey, H. Shea and P. Wurz, J Anal Atom Spectrom, 2017, DOI: 10.1039/C7JA00193B.

16. A. Riedo, A. Bieler, M. Neuland, M. Tulej and P. Wurz, $J$ Mass Spectrom, 2013, 48, 1-15.

17. V. Grimaudo, P. Moreno-Garcia, A. Riedo, S. Meyer, M. Tulej, M. B. Neuland, M. Mohos, C. Gutz, S. R. Waldvogek, P. Wurz and P. Broekmann, Analytical Chemistry, 2017, 89, 1632-1641.

18. V. Grimaudo, P. Moreno-Garcia, A. Riedo, M. B. Neuland, M. Tulej, P. Broekmann and P. Wurz, Anal Chem, 2015, 87, 2037-2041.

19. M. He, Y. Meng, S. Yan, W. Hang, W. Zhou and B. Huang, Anal Chem, 2017, 89, 565-570.

20. R. Wechsung, F. Hillenkamp, R. Kaufmann, R. Nitsche, E. Unsold and H. Vogt, Microsc Acta, 1978, 281-296.

21. N. C. Fenner and N. R. Daly, Rev Sci Instrum, 1966, 37, 1068\&. 
E. Bernal, L. P. Levine and J. F. Ready, Rev Sci Instrum, 1966 37, 938-\&.

23. Q. Yu, L. Z. Chen, R. F. Huang, W. Hang, J. He and B. L. Huang, Trac-Trend Anal Chem, 2009, 28, 1174-1185.

24. T. Dingle, B. W. Griffiths and J. C. Ruckman, Vacuum, 1981 31, 571-577.

25 J. F. J. Todd, Pure Appl Chem, 1991, 63, 1541-1566

26.

D. M. Lubman, Lasers and mass spectrometry, Oxford University Press, New York etc., 1990.

A. A. Sysoev and A. A. Sysoev, Eur J Mass Spectrom, 2002, 8, 213-232.

W. H. Guest, International Journal of Mass Spectrometry and Ion Processes, 1984, 60, 189-199.

D. M. Hercules, R. J. Day, K. Balasanmugam, T. A. Dang and C. P. Li, Analytical Chemistry, 1982, 54, A280-\&.

D. M. Hercules, Microchem J, 1988, 38, 3-23.

P. C. Lyons, D. M. Hercules, J. J. Morelli, G. A. Sellers, D. Mattern, C. L. Thompsonrizer, F. W. Brown and M. A. Millay, Int J Coal Geol, 1987, 7, 185-194.

L. Vanvaeck, J. Bennett, W. Lauwers, A. Vertes and R. Gijbels, Mikrochim Acta, 1990, 3, 283-303.

H. Vogt, H. J. Heinen, S. Meier and R. Wechsung, Fresen Z Anal Chem, 1981, 308, 195-200.

F. Bruynseels, H. Storms and R. Vangrieken, J Phys-Paris, 1984, 45, 785-788.

Y. M. Lin, Q. A. Yu, W. Hang and B. L. Huang, Spectrochim Acta B, 2010, 65, 871-883.

K. G. Bychik, A. S. Voloshko, E. E. Sil'nikov, A. A. Sysoev and A. A. Sysoev, J Anal Chem+, 2011, 66, 1455-1463.

T. Delgado, J. M. Vadillo and J. J. Laserna, J Anal Atom Spectrom, 2018, 33.

T. Stephan, R. Trappitsch, A. M. Davis, M. J. Pellin, D. Rost, M. R. Savina, R. Yokochi and N. Liu, Int J Mass Spectrom, 2016, 407, 1-15.

KORE, Mini-TOF-MS with Cassegrain Optics For Surface Laser Ablation Studies, https://www.kore.co.uk/1269.htm, (accessed 18.1.2019, 2019).

U. Rohner, J. A. Whitby, P. Wurz and S. Barabash, Rev Sci Instrum, 2004, 75, 1314-1322.

U. Rohner, J. A. Whitby and P. Wurz, Meas Sci Technol, 2003, 14, 2159-2164.

G. G. Managadze, A. A. Safronova, K. A. Luchnikov, E. A Vorobyova, N. S. Duxbury, P. Wurz, N. G. Managadze, A. E. Chumikov and R. K. Khamizov, Astrobiology, 2017, 17, 448458.

W. B. Brinckerhoff, G. G. Managadze, R. W. McEntire, A. F. Cheng and W. J. Green, Rev Sci Instrum, 2000, 71, 536-545. F. S. Anderson, J. Levine and T. J. Whitaker, Rapid Commun Mass Sp, 2015, 29, 191-204.

R. Wiesendanger, D. Wacey, M. Tulej, A. Neubeck, M. Ivarsson, V. Grimaudo, P. Moreno-Garcia, A. Cedeño López, A. Riedo and P. Wurz, Astrobiology, 2018, 10.1089/ast.2017.1780.

Z. Ma, R. N. Thompson, K. R. Lykke, M. J. Pellin and A. M. Davis, Rev Sci Instrum, 1995, 66, 3168-3176.

I. V. Veryovkin, W. F. Calaway, J. F. Moore, M. J. Pellin and D. S. Burnett, Nucl Instrum Meth B, 2004, 219, 473-479.

I. Kuznetsov, J. Filevich, F. Dong, M. Woolston, W. L. Chao, E. H. Anderson, E. R. Bernstein, D. C. Crick, J. J. Rocca and C. S. Menoni, Nat Commun, 2015, 6.
49.

T. Green, I. Kuznetsov, D. Willingham, B. E. Naes, G. C. Eiden, Z. H. Zhu, W. Chao, J. J. Rocca, C. S. Menoni and A. M. Duffin, J Anal Atom Spectrom, 2017, 32, 1092-1100.

50. V. Grimaudo, P. Moreno-Garcia, A. C. Lopez, A. Riedo, R. Wiesendanger, M. Tulej, C. Gruber, E. Lortscher, P. Wurz and P. Broekmann, Analytical Chemistry, 2018, 90, 26922700.

51. A. C. Lopez, V. Grimaudo, P. Moreno-Garcia, A. Riedo, M Tulej, R. Wiesendanger, P. Wurz and P. Broekmann, J Anal Atom Spectrom, 2018, 33, 283-293.

52. J. S. Becker, Inorganic mass spectrometry principles and applications, John Wiley, Chichester, 2007.

53. R. F. Huang, Y. M. Lin, L. F. Li, W. Hang, J. He and B. L. Huang, Analytical Chemistry, 2010, 82, 3077-3080.

54. I. V. Veryovkin, W. F. Calaway and M. J. Pellin, Nucl Instrum Meth A, 2004, 519, 353-362.

M. R. Savina, M. J. Pellin, C. E. Tripa, I. V. Veryovkin, W. F. Calaway and A. M. Davis, Geochim Cosmochim Ac, 2003, 67, 3215-3225.

F. S. Anderson, J. Levine and T. J. Whitaker, Rapid Commun Mass Sp, 2015, 29, 1457-1464.

57. M. Hassig, M. Libardoni, K. Mandt, G. Miller and R. Blase, Planet Space Sci, 2015, 117, 436-443.

H. Wollnik and M. Przewloka, International Journal of Mass Spectrometry and Ion Processes, 1990, 96, 267-274.

T. Sakurai, Y. Fujita, T. Matsuo, H. Matsuda, I. Katakuse and K. Miseki, International Journal of Mass Spectrometry and Ion Processes, 1985, 66, 283-290.

60. T. Sakurai, H. Nakabushi, T. Hiasa and K. Okanishi, Nucl Instrum Meth A, 1999, 427, 182-186.

61. D. M. Murphy, J Am Soc Mass Spectr, 2017, 28, 242-246.

62. A. A. Sysoev, A. V. Karpov, V. V. Milyaeva and A. A. Sysoev, Eur J Mass Spectrom, 2018, 24, 96-107.

63. R. Arevalo, L. Selliez, C. Briois, N. Carrasco, L. Thirkell, B. Cherville, F. Colin, B. Gaubicher, B. Farcy, X. Li and A. Makarov, Rapid Commun Mass Sp, 2018, 32, 1875-1886.

64. R. P. Schmid and C. Weickhardt, Int J Mass Spectrom, 2001, 206, 181-190.

65. S. Scherer, K. Altwegg, H. Balsiger, J. Fischer, A. Jackel, A Korth, M. Mildner, D. Piazza, H. Reme and P. Wurz, Int $J$ Mass Spectrom, 2006, 251, 73-81.

66. B. C. Zhang, M. H. He, W. Hang and B. L. Huang, Analytical Chemistry, 2013, 85, 4507-4511.

67. L. F. Li, B. C. Zhang, R. F. Huang, W. Hang, J. He and B. L. Huang, Analytical Chemistry, 2010, 82, 1949-1953.

68. R. Wiesendanger, M. Tulej, V. Grimaudo, A. Cedeño-López, R. Lukmanov, A. Riedo and P. Wurz, Journal of Chemometrics, 0, e3081.

69. M. Tulej, A. Neubeck, M. Ivarsson, A. Riedo, M. B. Neuland, S. Meyer and P. Wurz, Astrobiology, 2015, 15, 669-682.

70. M. Tulej, M. lakovleva, I. Leya and P. Wurz, Analytical and Bioanalytical Chemistry, 2011, 399, 2185-2200.

71. A. Miotello, Laser-surface interactions for new materials production Tailoring structure and properties, Springer, Heidelberg, 2010.

72.

B. Toftmann and J. Schou, Appl Phys a-Mater, 2013, 112, 197-202.

73. H. Varel, M. Wahmer, A. Rosenfeld, D. Ashkenasi and E. E. B. Campbell, Appl Surf Sci, 1998, 127, 128-133.

74. V. Grimaudo, P. Moreno-Garcia, A. Riedo, M. B. Neuland, M. Tulej, P. Broekmann and P. Wurz, Analytical Chemistry, 2015, 87, 2037-2041. 
75. X. H. Wang, S. D. Zhang, X. L. Cheng, E. Y. Zhu, W. Hang and B. L. Huang, Spectrochim Acta B, 2014, 99, 101-114.

76. T. Donnelly, J. G. Lunney, S. Amoruso, R. Bruzzese and X. Wang, International Symposium on High Power Laser Ablation 2010, 2010, 1278, 643-+.

77. X. C. Ni, K. K. Anoop, X. Wang, D. Paparo, S. Amoruso and R. Bruzzese, Appl Phys a-Mater, 2014, 117, 111-115.

78. B. A. Mamyrin, V. I. Karataev, D. V. Shmikk and V. A. Zagulin, Zh Eksp Teor Fiz+, 1973, 64, 82-89.

79. R. G. Fausch, P. Wurz, D. Lasi, M. Tulej, M. Cabane, C. Szopa, A. Sapgir and M. Gerasimov, 2018.

80. D. Abplanalp, P. Wurz, L. Huber, I. Leya, E. Kopp, U. Rohner, M. Wieser, L. Kalla and S. Barabash, Advances in Space Research, 2009, 44, 870-878.

81. S. Meyer, A. Riedo, M. B. Neuland, M. Tulej and P. Wurz, Journal of Mass Spectrometry, 2017, 52, 580-590.

82. P. L. Pryor, J Opt Soc Am, 1950, 40, 801-801.

83. P. Moreno-Garcia, V. Grimaudo, A. Riedo, A. Cedeno Lopez, R. Wiesendanger, M. Tulej, C. Gruber, E. Lortscher, P. Wurz and P. Broekmann, Anal Chem, 2018, 90, 6666-6674.

84. D. S. Finn, Z. B. Lin, J. Kleinert, M. J. Darwin and H. B. Zhang, J Laser Appl, 2015, 27.

85. D. L. Donoho and I. M. Johnstone, Biometrika, 1994, 81, 425-455.

86. D. L. Donoho, leee T Inform Theory, 1995, 41, 613-627.

87. M. Berglund and M. E. Wieser, Pure Appl Chem, 2011, 83, 397-410.

88. R. B. Marinenko, Heinrich, K. F. J., and Ruegg, F. C., MicroHomogenity Studies of NBS Standard Reference Materials, NBS Research Materials and Ohter Related Samples, National Bureau of Standards, Boulder, CO, 1979.

89. A. Riedo, M. Tulej, U. Rohner and P. Wurz, Rev Sci Instrum, 2017, 88, 045114.

90. G. Wells, H. Prest, C.W. Russ. Technical note from Agilent Technologies: signal, noise and detection limits in mass spectrometry, 5990-7651E, 3 May 32011. 